\title{
ASTRONOMICAL OBSERVATORIES: FROM THE PREHISTORY TO THE XVIII CENTURY
}

\author{
M. A. Castro Tirado ${ }^{1,2}$
}

\begin{abstract}
We describe the development of astronomical observatories since the prehistory until the late XVIII century, and how an evolution of several millennia took us from very elementary structures to buildings hosting telescopes and domes in which science was the fundamental, but not the only motivation.

\section{RESUMEN}

Describimos el desarrollo de observatorios astronómicos desde la prehistoria hasta finales del siglo XVIII, y cómo la evolución de varios milenios ha pasado de estructuras elementales a edificios albergando telescopios y cúpulas en los cuales la ciencia era fundamental, pero no la única motivación.
\end{abstract}

Key Words: history and philosophy of astronomy - telescopes

\section{INTRODUCTION}

Astronomy originates from the primitive relationship between man and nature. Especially with its most unreachable component: the sky.

In antiquity, some of the stars were considered gods and the sky was their residence. Therefore there was a strong connection between astronomy and religion that only began to diverge a few centuries ago. In addition to this spiritual sense, the beginnings of this science point to the instrumentalization of the firmament as a system of measurement of time periods and as a means of orientation.

Beyond the numerous artistic representations of the Sun, Moon and stars that have been found, or of the written testimonies that have lasted in time, there is evidence of this astronomical preference through the incidence of some celestial phenomena in the arrangement of certain constructions or archaeological sites.

In any case, although certain vestiges of ancient civilizations evidenced an outstanding impact of astronomy in its culture through its architecture or urbanism, it will be the proto-observatories, understood as places conceived exprofeso to be the ones destined, in some way, to the study of the heavens. Those that stand out as points of interest to know the way in which these people approached the study of this science.

These spaces will be where contemporary astronomical observatories have their deepest roots.

\footnotetext{
${ }^{1}$ Instituto de Astrofísica de Andalucía (IAA-CSIC), Granada, Spain.

${ }^{2}$ Departamento de Ingeniería de Sistemas y Automática (Unidad Asociada al CSIC), Escuela de Ingeniería Industrial, Universidad de Málaga, Málaga, Spain.
}

Dreyer (1953), Hall (1983), Magli (2009), Sayili (1960) and Thurston (1994) have been consulted for the present review.

\section{STONE AGE}

The earliest set of recordings in Prehistory to date is the Goseck Circle (Figure 1). It consists of a neolithic structure located in Germany dating about 7000 years old. It is a solar observatory of annular plant, with an inner radius of 48 meters and an outer radius of 82 . It is composed of four concentric rings, being from outside to inside, the first a mound, the second a moat and finally two palisades. These crowns have three discontinuities aligned radially and focused respectively towards the north, southeast and southwest. These openings are oriented towards the sunrise and sunset on the winter solstice.

Of a similar chronological period is the megalithic set of Nabta Playa, in Egypt, to the south of Cairo. It presents a circular composition of almost thirty perimeter stones marking doors with larger pairs and containing some inner clusters.

Although this place could accommodate some religious uses associated with the nomadic cultures of that context, an archaeoastronomical character is recognised. Through the study of the firmament the circle would acquire functions of calendar, marking two of its doors an alignment with the summer solstice, and point of orientation in the desert.

\section{PROTOHISTORY}

Sometime later, around 3000 BC Carahunge (also known as Zorats Karer) can be situated. Located in 


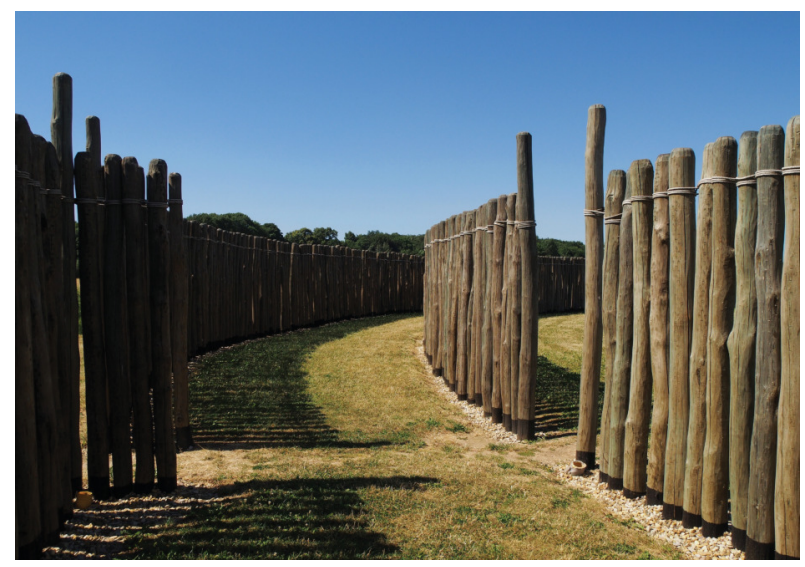

Fig. 1. Reconstruction of Goseck Circle.

Armenia is this composition of more than one hundred stones arranged as menhirs of between one and three meters height with plant in form of oval ring.

The main singularity of this formation is that part of the rocks that compose it have a perforation of between 5 and $20 \mathrm{~cm}$ diameter that crosses them and that, according to what has been seen, point towards significant events as the Sun at dawn and dusk in the winter solstice.

Between 3600 and $2500 \mathrm{BC}$ the megalithic temple of Mnajdra is erected, located on the south coast of Malta. The vestiges that have survived until the present day consist of three constructions of limestone, that although they are not connected they are grouped around a place between the remains of a major complex but scarcely conserved.

Beyond the discovery of signs that point to a religious character of the constructions, there are arguments that support the idea that identifies the southernmost temple as an observatory dedicated to the study of heaven and time. In particular its orientation and configuration cause the sunrise of the summer and winter solstices passing through the main door and illuminate the megaliths located respectively left and right of the door. Also, at the spring and autumn equinoxes, at dawn, the Sun enters through the same door crossing the main nave orthogonally to the altar.

The famous Stonehenge (Figure 2) is located in England, west of London, and its construction extends from 3100 to $1600 \mathrm{BC}$. Although an important megalithic structure remains, a considerable part of the initial composition has been lost. However, the remains are sufficient so that the studies have been able to define its original configuration.

It displays a circular plant of about 30 meters of diameter conformed by concentric constructions.

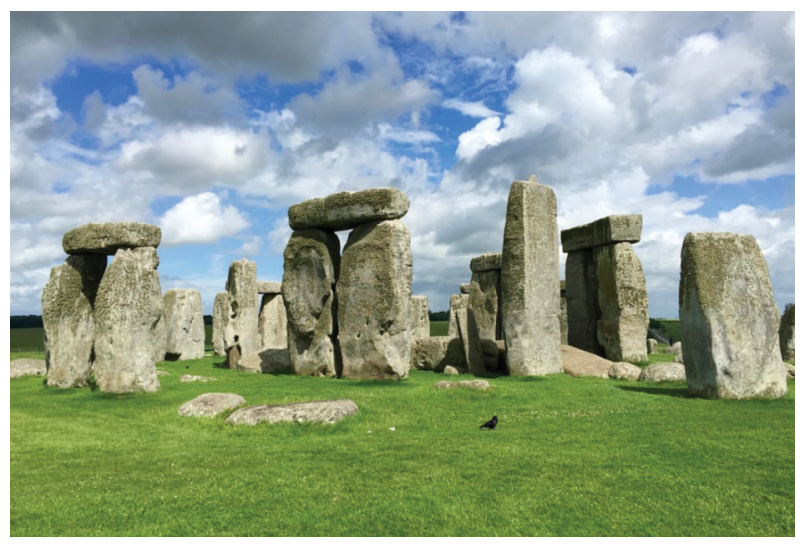

Fig. 2. Stonehenge.

The outer perimeter was delimited by a series of vertical blocks that supported lintels forming a closed ring. Concentrically there was a circumference of smaller monoliths that surrounded five pairs of large stone blocks forming doors. The interior was occupied by a set of menhirs.

The alignments that occur in Stonehenge through its gates during the sunrise and sunset during the summer and winter solstices are obviously intentional. This shows a certain degree of astronomical knowledge on the part of its constructors and denotes its function as observatory and calendar beyond other ritual or religious uses associated to these facts.

In any case, the astronomical functions of these structures were quite limited. It is even possible that the scientifically valuable activity can be limited to the determination of the positions and orientation of the elements of the set. A study of the sky is required with systematic and intentional observations compared to these reference points that allow to establish a pattern from which to erect these constructions. Once the device is 'calibrated', using it is not much more scientific than looking at the hands of a watch.

\section{ANCIENT HISTORY}

The above mentioned cases allude to primitive civilizations, with a low degree of technification and even, frequently, with nomadic habits. This makes simple the identification of their constructions.

However, the evolution towards more complex cultures makes this difficult. This is so because the more advanced civilizations tend to create settlements with multiple buildings in which it is unnecessary to reach the degree of specialization needed for the function of observation as such. Since no 


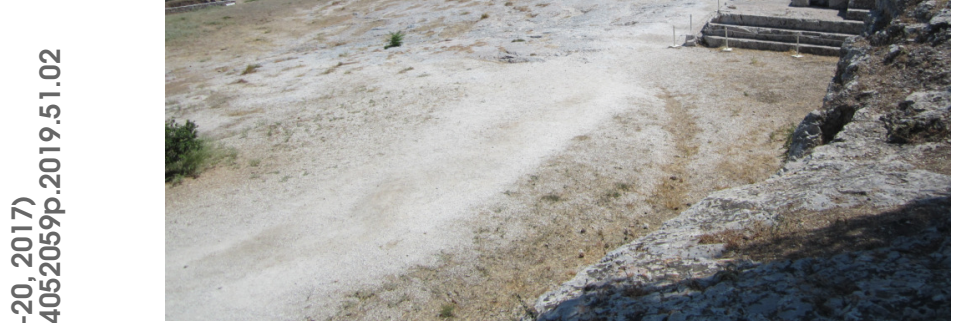

Fig. 3. The Pnyx at Athens.

scientific instruments had been developed that required demanding conditions, this recognition of the sky could be carried out with the naked eye in almost any construction or even in the vicinity of these open skies.

In the case of Mesopotamian astronomy, although there is certainty of its development, there is little knowledge about the instruments used for the study of the sky, leaving only a few items in museums. So it is very difficult to clarify where the observatories of this civilization were located, although it is possible that these were incorporated as suitable spaces for that objective in other buildings. Or even that certain rooms or areas, such as the terraces of the ziggurats, were used in a timely manner for this purpose.

Precisely, according to some writers of the antiquity such as Herodoto, Plinio or Diodoro, the Tower of Babel was used for the astronomical observation of stars.

Something similar happens with regard to Greek culture in the Classical antiquity period, with an important tradition in astronomy and with a crucial role in the history of this science. There is no evidence of built observatories as such.

Nevertheless, there are vestiges of some places used for the study of the sky, like the Pnyx in Athens, which dates from sixth century BC (Figure 3). Its astronomical use has been recorded since at least the fifth century BC. Even if this place was not only destined to this function, it was destined to the political assembly, as its suitable characteristics propitiated the new use. Formed by a semicircular flat platform in an elevated position and with a wide view of the sky, it ended up receiving instruments of astronomical study (the heliotrope of Meton).

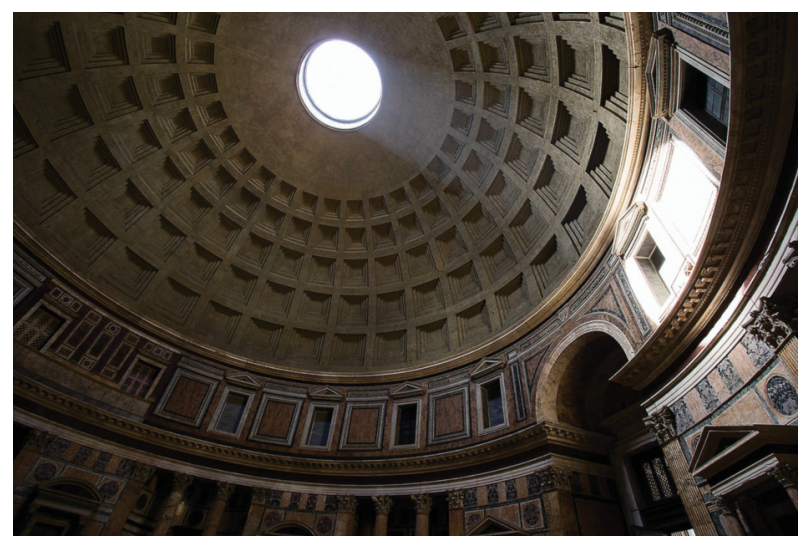

Fig. 4. The Sun through the Pantheon.

Regarding the Roman influence, although its relevance is smaller than the one inherited from other civilizations, it is possible to stand out points of celestial investigation the Field of Mars of Rome, where the solar clock of Augustus was located, and the Pantheon, constructed during the second century, in which, thanks to its oculus, solar studies were carried out. Its design and orientation make it possible for a beam of light during the zenith of the equinox to cross the building from the oculus to the portico, through a lattice-work over the main door, indicating that event (Figure 4).

\section{POST-CLASSICAL HISTORY}

At the other end of the world is Cheomseongdae, in South Korea. It is the oldest astronomical construction of the Asian continent. It consists of a bottle like shape tower, about ten meters high and about six meters diameter, composed of granite masonry (Figure 5). It is kept in a great state of preservation.

The main function of this construction is to raise the astronomical instruments and their user over the floor. The user accesses by means of a ladder to an entrance located at the middle of its height, through which it passes into the interior space: a cylinder hollow with a second ladder to reach the upper opening.

The main historical contribution of China to astronomical constructions is the Great Dengfeng Observatory, built around 1279. It is a tower $\sim 10$ meters high, with leaning walls of stone and brick, whose peak was known as the "platform of observation of stars". It has two rooms at the top for the study of the sky from a sheltered space and a large gnomon that is part of the structure itself.

Even without receiving any influence from foreign civilizations, the different cultures of the American continent also present constructions related to the 


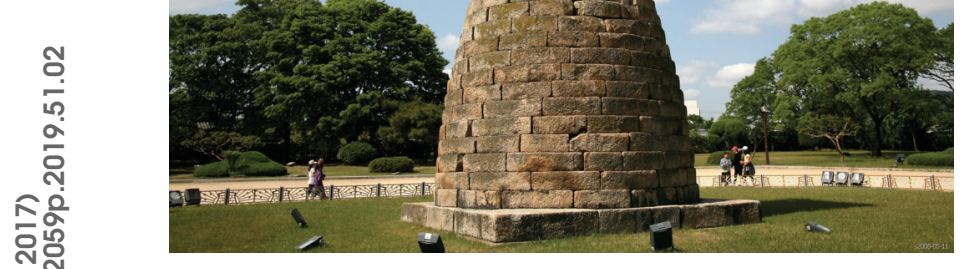

Fig. 5. Cheomseongdae.

study of the sky. Among these cultures the Maya stands out, especially, for its advanced astronomy and for the complexity of its observatories.

Of these astronomical structures can be highlighted the Caracol in Chichen Itza: a circular plant construction of 12.5 meters high with an internal spiral staircase to access the top that gives name to the observatory. Built between the 8th and 9th centuries, it was used to set astronomical observation points and to follow the movements of the planet Venus.

Coinciding with the beginning of the Middle Ages, begins a time of cultural darkness in the Western world that would not escape astronomy. During this period, Islamic culture will play an essential role in the history of this science, preserving the vast Greek knowledge of this subject, and incorporating its own inquiries, which would be recovered by the west during the thirteenth century, especially by the School of Translators of Toledo.

For the Islamic people astronomy has an important value, since the Koran provides numerous references to the Sun, the Moon or the stars, influencing their daily life through religious customs such as the hours of prayer or the beginning of Ramadan.

The astronomical tradition is reflected in the methodical study of the firmament in temporary observation posts.

The construction of the observatory in Maragha, northwest of Iran, is part of the period that represents the highest point of the evolution of the Islamic observatory, during the thirteenth century, when they move from these temporary posts to a scientific institution located in a building.

The main building was a twenty-two meters diameter circular tower. The central space was aligned from north to south with the meridian, containing a mural quadrant that occupies the entire central vol-

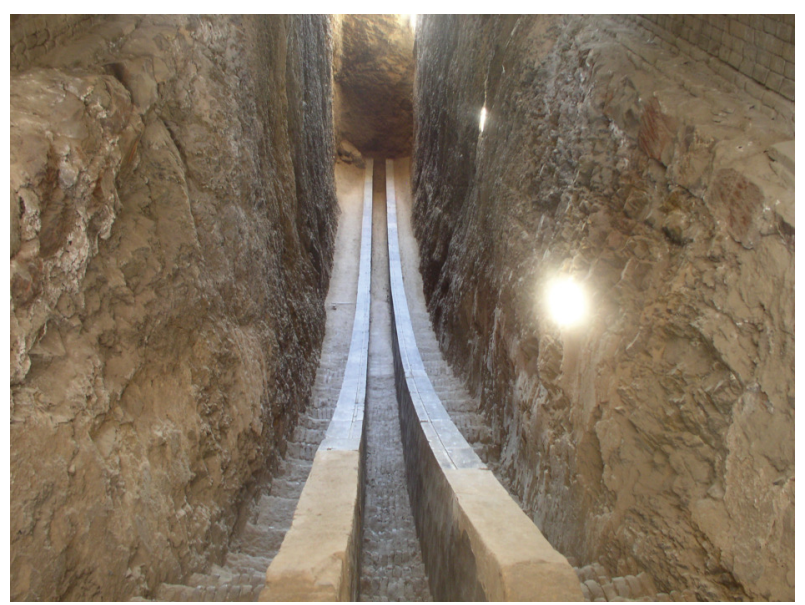

Fig. 6. Remnants of the observatory of Samarkand.

ume. To the sides of this space appeared perpendicularly rooms of smaller size to house the library and instruments of observation and working tables. Above this tower there was a small dome with an oculus to measure the movements of the sun.

The advances made in Maragha had their influence in later astronomical constructions. Thus the observatory is conceived as a collaborative institution in which several researchers developed a particular study during a certain period of time and with homogeneous working conditions.

This was reflected in the observatories of Istanbul or Samarkand (Figure 6), with a design similar to that of Maragha.

\section{MODERN HISTORY}

The first references to an European observatory point towards Nürnberg, where astronomer and mathematician Johannes Müller von Königsberg carried out his observations at the end of the fifteenth century. Shortly after, the observations developed by Copernicus in Frauenburg, Ermland and Allestein during the first half of the sixteenth century are recorded.

However, it appears that these observations occurred at various locations in the open field.

In any case, there is no evidence to suggest that any of these astronomers had an observatory. Furthermore, they lacked an observatory as such. Their work paved the way for the subsequent projects of Wilhelm IV and Tycho Brahe.

\subsection{The first modern observatory}

In the Landgraviate of Hesse-Kassel, in 1560, Landgrave Wilhelm IV, fascinated by this science, orders to construct a balcony-like platform at his urban palace. This viewpoint aimed at studying the 


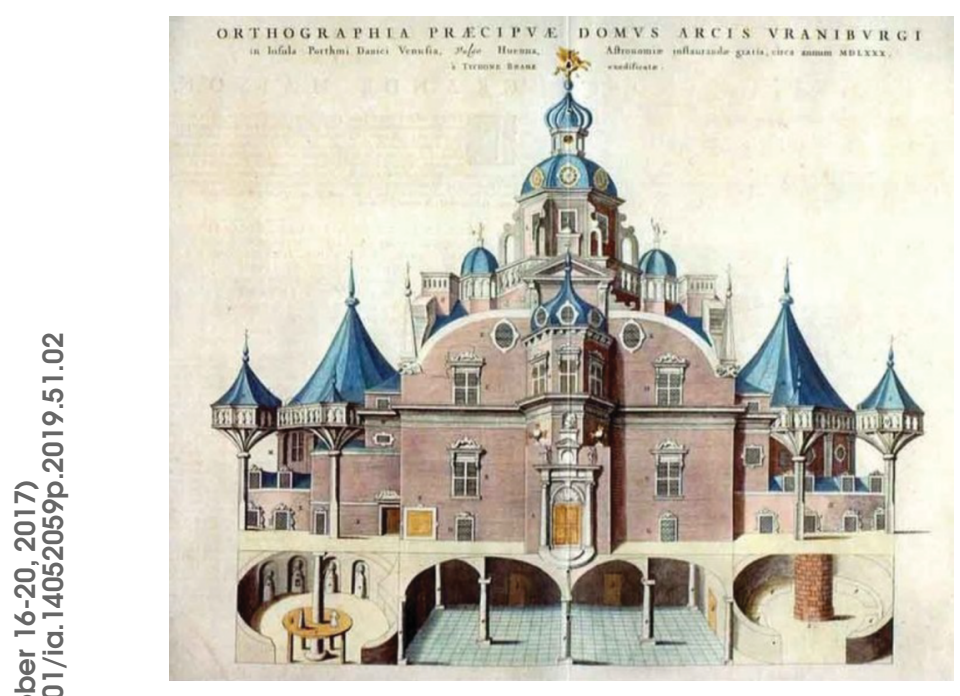

Fig. 7. Representation of Uraniborg by Tycho Brahe.

sky by means of astronomical instruments. Although initially the landgrave itself would make the observations, soon other astronomers would be incorporated for the development of a permanent investigation.

Even if this platform I just mentioned may be considered by some authors as the first observatory of the Modern Age, it does not represent any substantial qualitative advance compared to those of previous periods, resulting, to a large extent, from improvisation.

However, the relevance of the Hesse-Kassel observatory is no smaller, since it would serve as a catalyst for the evolution of this building typology. In 1575 Tycho Brahe visited the Landgrave Wilhelm's facilities and, a few months later, inspired by that trip, began to build in Denmark which, now, will be the first modern observatory.

Tycho counted on several architects for the design of Uraniborg, in which its enormous relevance does not lie on its stylistic features but on its formfunctional conception.

So far, the instruments of observation were simply placed on platforms chosen with certain criterion.

Astronomer Giovanni Domenico Cassini would point Uraniborg as the birthplace of modern astronomy.

At Uraniborg stands a building that responds and conforms to a specific program of uses. The area for astronomy rises to the platforms at the top, allowing the instruments to view the sky and freeing them from obstacles. The alchemy is carried out on the basement, closest to the ground to take advantage of the heat of the fires that heat the building from below up. In the middle part, there is a study

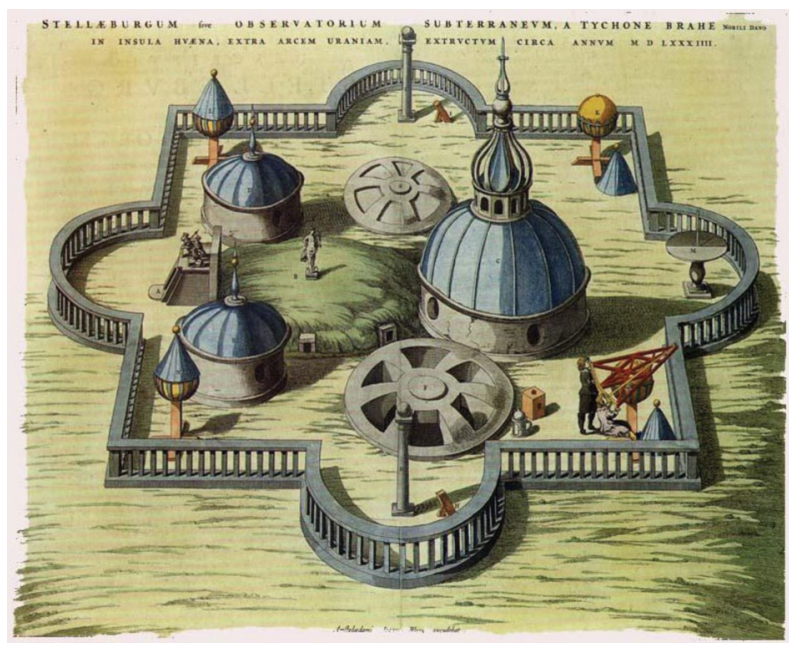

Fig. 8. Representation of Stjerneborg by Tycho Brahe.

area, service spaces and residential uses (Figure 7).

The project takes into account the segregation of uses according to their compatibility and their arrangement according to their needs. It also considers issues as important as lighting, heating and ventilation.

Nevertheless, the project has some flaws in its concept, with instability of observation platforms being particularly important.

This research center will have such an impact that it will become a reference to be imitated by later European observatories, if not in its appearance, at least in its conception; and will mark the future of the later observatories in which the relationship between science and architecture will be essential.

Only a few years after completing Uraniborg, Tycho opened a new observatory in the vicinity of the first one with the intention of solving stability problems: Stjerneborg (Figure 8).

For this, a partially buried construction is used in which the observation instruments are installed on firm pier.

Stjerneborg groups a series of specialized rooms for each particular instrument, with all of them around a central workspace.

These spaces represent their singularity in a specific type of cover (domes, demountable slabs ...) and in the definition of their floors, which adapt to the working conditions for each instrument.

It will not be until the late 17th century when other astronomical observatories that have the institutional entity presented by those of Tycho Brahe begin to appear.

Meanwhile, the evolution of this science in a branched way entailed different techniques of study 
that, sometimes, moved away from a direct observation. This is the case with the camera obscura.

Despite lacking the right architecture for observation, Renaissance astronomers maintained their research performance in line with their significant operational limitations. That is, by accommodating existing constructions and spaces to an astronomical function.

Tycho Brahe, dedicates a section of his work Astronomiae Instauratae Mechanica to an architectural reading of these buildings, in which he emphasizes the importance of the place of implantation or the fact that the design responds to the practical function.

Some years later, Juan Caramuel, a cistersian monk, will dedicate an article from his treatise Arquitectura Civil Recta y Oblicua to the same question. However, his perspective, while maintaining a functional vision, is much more aseptic and idealized than that of Tycho, given that Caramuel, not being an astronomer, is not involved and does not devote so much attention to the user of the building.

\subsection{The design taking into account the use of the telescope}

The incorporation of the telescope as main tool of work would mark the future of the design of astronomical observatories.

However, since the first telescopes used to be of small size and quite manageable, many astronomers concluded that they could make their observations from the windows, balconies, terraces or gardens of their own residence. That is why, during this period, the development of the observatories goes towards simple structures or, directly, to additions on preexisting buildings where platforms are installed from which the sky is better visualized.

For example, in Holland in 1633 the Observatory of the University of Leiden was founded, building an extension on the existing roof and being the oldest in Europe integrated into a public institution.

Throughout the seventeenth century, various structures were established with an observatory vocation throughout Central Europe, highlighting the Rundetårn in København: a case in which, despite the greater architectural complexity, the astronomical bases remain very elementary (Figure 9).

It is a tower that has a helical upward sloping development around a hollow cylindrical core. At the top, more than forty meters high, the ramp opens onto a slightly inclined platform with views of three hundred and sixty degrees around the nucleus.

In spite of the apparently ideal conditions of the platform, it lacked the necessary characteristics for

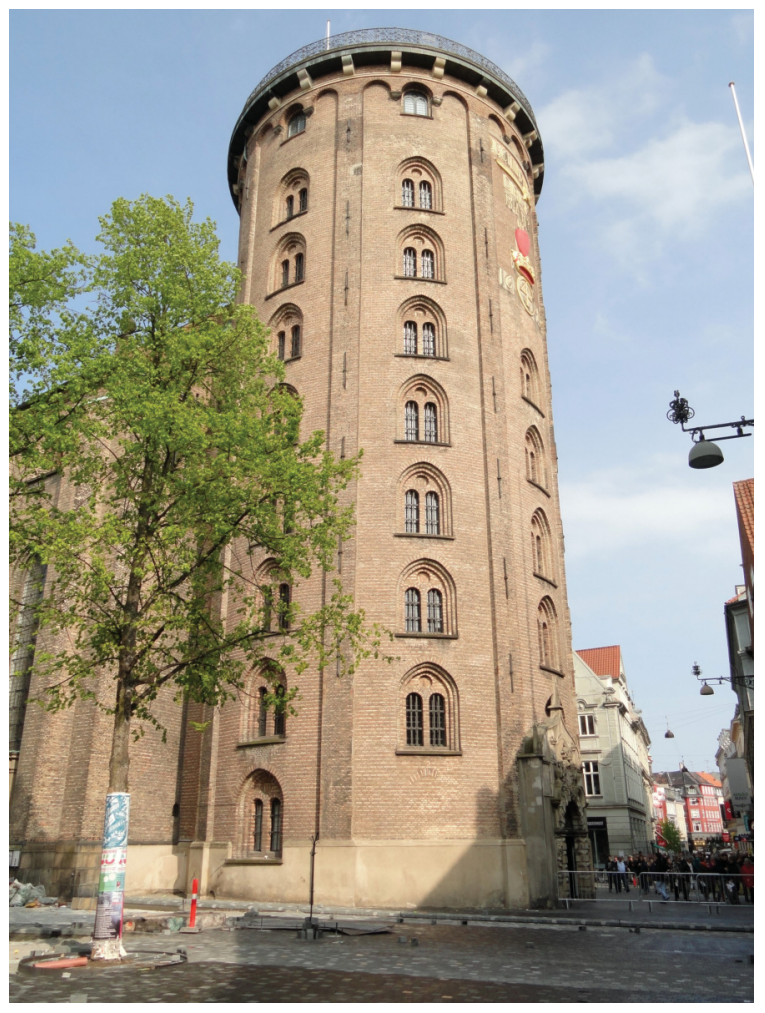

Fig. 9. Rundetårn in Copenhagen.

a good observatory, as its director Ole Rømer would recognize.

The growth of the telescopes for the sake of greater magnification gave rise to stability problems for pointing objects in the sky, which was solved in the instruments of zenith in which the telescopes were installed vertically attached to some type of structure which ensured its fixity and balance.

This is the case of Robert Hooke's telescope at Gresham College to measure parallax. Faced with the need for an installation in a high and narrow, undisturbed space, for an extended period of time, he chose to accommodate it in his own residence. This was mounted vertically, through the deck and a floor, to make the observations from the lower level. In addition it incorporated a system that allowed its cover and shelter in bad weather or when it was not in use.

On the other hand, towards the second half of the century, began a race to construct telescopes of greater length, reason why aerial models were developed increasingly longer and more complicated to use. These instruments became so long that they could no longer fit inside buildings. In addition to the problem of obtaining a location in which to install them, their exposure made difficult their han- 


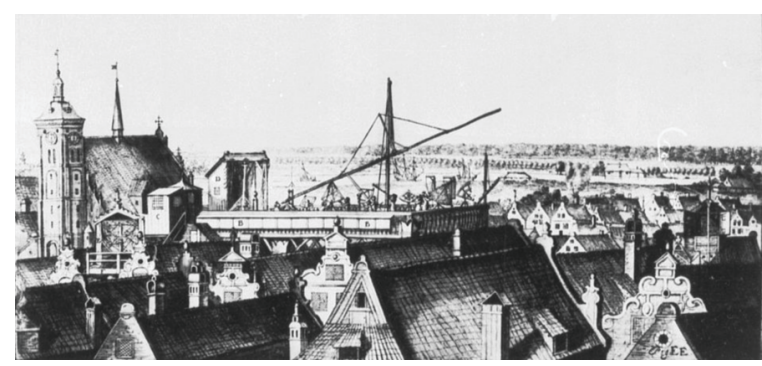

Fig. 10. Johannes Hevel's rooftop platform.

dling and the tasks of measurement or data collection necessary for the investigation.

Take for example Robert Hooke's twelve-meterlong telescope at Gresham College in London or Christian Huygens' devoid of casing models.

Another case is the Johannes Hevel, who installed a platform on the roof of his home from which to make observations with his telescopes. He developed increasingly long instruments until he got one so large that he was forced to take it out in the open sky to the outskirts of the city, installing it on a pole suspended by several braces (Figure 10).

Observation with a telescope of these dimensions should not have been simple, on the contrary. A small group of collaborators was necessary to transfer, erect and assemble perfectly the sections of the field telescope, as well as for its handling during the observations.

To the complex operation of the telescope, due to its size, we must add the difficulties of working exposed to the elements.

Taking another step, Hevel presents in his Machinae Coelestis of 1679 a theoretical project for an observatory. The design is based on the superposition of two well-differentiated elements: a great platform to be able to make observations with several telescopes simultaneously and a rotating tower to suspend them.

Rømer, director of the Rundetårn, moves the observations to the architectural framework of his own house, where he occupies a south-facing room for such use, where he installs his "machina domestica".

A particular observatory based on a meridian transit telescope adapted to the architectural circumstances existing in his dwelling, where the conditions of stability and climatization are favorable.

To what extent did the architecture condition its observations? That is to say, did Røemer design a device that, by pure chance, could adapt to the space that already had or devised his "domestic machine" to adapt to the architecture of his house? The second possibility seems more likely, less random.

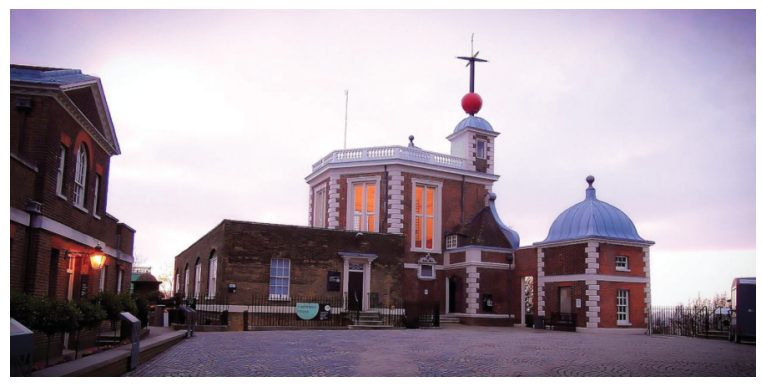

Fig. 11. The Flamsteed House at the Greenwich Observatory.

The Paris Observatory was born as a response to the demands of the newly established Académie des Sciences, in a context of full boil Illustration, whose aim was to symbolize greatness and renown through knowledge.

The design was commissioned by a famous architect and member of the Academy: Claude Perrault. The project developed by him had a monumental character and looked more like a palace than a building designed for astronomy. Despite Perrault's good intentions, the initial proposal had many drawbacks, a result of the lack of knowledge and lack of astronomical experience.

Luckily, during the construction, Giovanni Domenico Cassini joined the project as an astronomy consultant, to contribute with his knowledge.

From this team resulted the design changes that ended up giving rise to a building that hosted both scientific activity and other service functions. In addition, it was full of successes and innovations that would be transferred to observatories and even other constructive typologies, being especially important the use of the flat roof.

\subsection{The introduction of the dome}

The Greenwich Observatory. In this case, the development of maritime trade routes and military dominance of the seas causes the observatory's main objective to be functional and practical: to develop navigation.

Sir Christopher Wren, professor of astronomy and architect, would be named for this task. And he also collaborated with the astronomers Flamsteed and Hooke.

Thanks to this team, a building was erected where the science and the quartermaster or services of support of this one coexisted (Figure 11).

Although the project presented all the necessary conditions to develop the astronomical activity in an appropriate way according to the methods of its time, the economic restrictions involved the use of an 


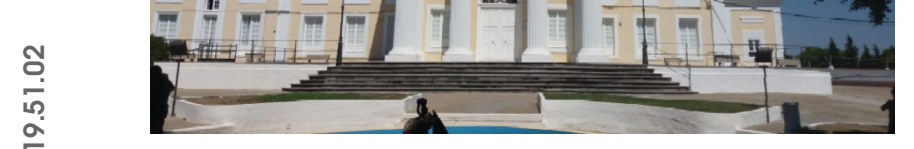

Fig. 12. The Spanish Royal Navy Observatory in San Fernando.

existing structure, which prevented its proper orientation.

This fact and the very development of science, were phasing out the observatory, which was adapted by modifications and extensions over time.

The Observatory of San Fernando (Cádiz) in Spain has a purpose homologous to that of its British predecessor: to improve navigation. In this case, in addition, the institution is linked to the Marine Guard Academy, with a dual function: research and training (Figure 12).

Initially the Observatory is installed in an existing construction: the Castle of the Villa. It occupies a battered tower where, despite occupying a privileged position, its operation is complicated and inefficient.

Years later it would end up moving to a building specifically for the Observatory, designed by the Marquis of Ureña, which would include spaces for astronomy and calculations derived from observation, and other zones of services for occupants and scientific activity.

The Madrid Observatory arises from the contagion of Illustration thought that spreads throughout Europe as an institution of knowledge and prestige. For this reason, its location is taken into account, in the extension of a cultural axis with the Prado Museum and the Botanical Garden.

The project is entrusted to the architect Juan de Villanueva who, beyond satisfying the functional requirements (both astronomical and service) takes into consideration the position that the building will occupy, high and visible from the entire urban front (Figure 13).

As a result of this, the observatory is developed as a monumental composition in which the whole environment will be part of the project, including a body of entrance and the landscape treatment of the surroundings.

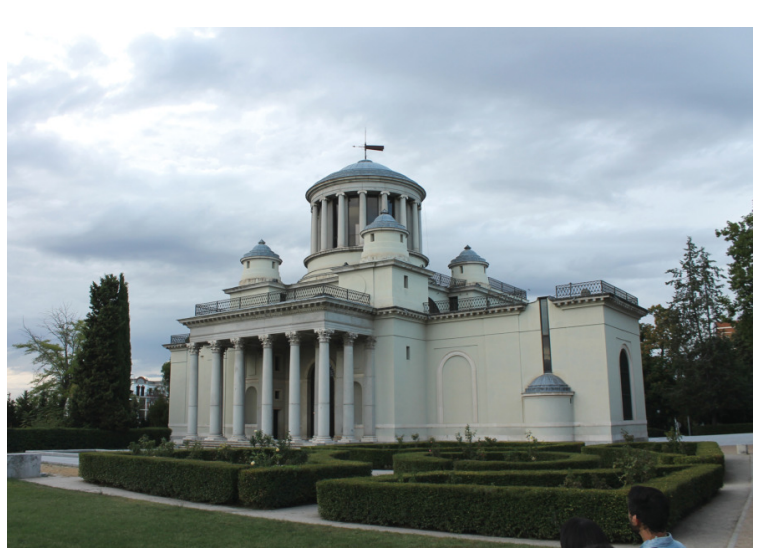

Fig. 13. Madrid Royal Observatory.

\section{CONCLUSIONS}

We have described the development of astronomical observatories since the prehistory until the late XVIII century. At this stage, I would like to point out how an evolution of several millennia took us from very elementary structures to buildings in which science was the fundamental, but not the only motivation.

These are the key points which have been reached:

- The best possible conditions for the development of astronomy.

- A compromise between astronomical activity and the needs of its users.

- A balance between functionality and comfort.

- The inclusion of movement and adaptation to climatic and lighting conditions.

- An accommodation to existing technology.

- The consideration of the environment, position and landscape.

Acknowledgements: We are grateful to Junta de Andalucia grant under project TIC-2839.

\section{REFERENCES}

Dreyer, J. L. E. 1953, A History of Astronomy, from Thales to Kepler, (New York, NY: Dover)

Hall, A. R. 1983, The Revolution in Science, 1500-1700, (New York, NY: Longman, Green and Co.)

Magli, G. 2009, Mysteries and Discoveries of Archaeoastronomy, (New York, NY: Springer Verlag)

Sayili, A. 1960, The Observatory in Islam and its place in the general history of astronomy, (Ankara, Turkey)

Thurston, H. 1994, Early Astronomy, (New York, NY: Springer Verlag) 